\title{
Malnutrition, stress and immunodepression: their interrelationship with fungal infections
}

\author{
I. Cesaroni, F. Negro and M. Guerrini \\ LABBCE, La Plata, Argentina
}

Prolonged stress, malnutrition and inappropriate nutrition represent $90 \%$ of the causes of immunodepression in subjects without basal pathologies, and are key to the entry into epithelia and endothelia of fungal species coexisting in the environment, as they provide by immunological deficit an optimal and permissive host. Both persistent emotional and nutritional disorders converge in metabolic stress, with hyperproduction of free radicals and an increase in cAMP. On the one hand, excessive production of free radicals, not neutralized because of the antioxidant deficit, impacts on the skin, thus generating tissue microlesions. On the other hand, cAMP accumulation produced by energy consumption associated with stress acts as a negative modulator of cellular immunity that decreases the activity of T-cells and macrophages. Consequently, there is a decrease in levels of IL, interferon- $\gamma$, TNF $\alpha$ and Ig, providing optimal conditions for the entry of opportunistic pathogens. These outcomes are manifest in numerous cases as severe surface mycoses, and in some cases as profound and systemic mycoses.

When patients with mycosis in different zones $(n>2400)$ were analysed $60 \%$ showed onychomycosis (infection of finger and toenails) and $40 \%$ a dermal mycosis, some with genetic immunological restrictions similar to psoriasis ${ }^{(1)}$ and vitiligo (20\% of those studied). In the remainder of cases mycosis was coincident with immunodepression in patients such as badly-nourished and/or malnourished children and elderly adults, breast-feeding mothers, postnatal mothers, alcohol- and drug-dependent patients, patients undergoing prolonged treatment with quimiotherapeutics, radiotherapeutics and corticosteroids, with emotional stress and other less-frequent cases.

Analysis of observed causes and outcomes with an unexpected finding has led to the postulation of a treatment model based on 350 patients with psoriasis participating in a randomised double-blind study that included a natural probiotic solution based on four species of Lactobacillus associated with Saccharomyces cerevisiae in oat solution. This product is a leading probiotic solution because of its topical and oral use. Periodic consumption of the probiotic ${ }^{(2)}$ allows recovery of the immunomodulating action of the intestinal mucosa, which contains $70 \%$ of the body's immune cells. Furthermore, its topical application facilitates indispensable degradation of the hyperkeratosic micaceous plaque stimulated by fungal toxins at the level of dermal receptors and growth factors, Rhodotorula and Malassezia ${ }^{(3)}$ being the effectors most involved.

Antifungal agents used in the form of cream, lotion or pills are selected according to results obtained by culture and antifungal tests of isolated species following the norms of the Clinical and Laboratory Standards Institute for antifungal tablets. The therapy was complemented with a diet based on fruits, vegetables, food containing $n-3, n-6$ and $n-9$ PUFA and a supplement of minerals and antioxidants formulated to provide rapid reversal of acute cases. Some cases also required psychological support.

Compared with patients treated with placebo, patients treated with the probiotic and antifungal agent showed overall improvement in their lesions. As the causes of immunodepression were controlled, mycotic growth could be stopped and the immunological deficit reversed by dosing with $\operatorname{IgA}$. In turn, this focused treatment allowed a specific action against the invasive fungal agent that resulted in favourable responses, decreasing not only the cost but also the duration of the treatment.

1. Baroni A, Paoletti I, Ruocco E, Agozzino M, Tufano MA \& Donnarumma G (2004) J Cutan Pathol 31, 35-42.

2. Weston S, Halbert A, Richmond P \& Prescott SL (2005) Arch Dis Child 90, 892-897.

3. Baroni A, Orlando M, Donnarumma G, Farro P et al. (2006) Arch Dermatol Res 297, 280-288. 